\title{
Repercussão da qualidade de vida de mulheres com câncer de mama durante a pandemia da COVID-19
}

\author{
Repercussion on the quality of life of women with cancer during the COVID-19 pandemic \\ Repercusión en la calidad de vida de las mujeres con cáncer de mama durante la pandemia
}

COVID-19

Maria Samara Da Silva

ORCID: https://orcid.org/0000-0001-6987-1224 Universidade Estadual do Piauí, Brasil E-mail: mariasamara2v@gmail.com

Amanda Célis Brandão Vieira ORCID: https://orcid.org/0000-0002-1494-8973 Faculdade Inspirar, Brasil E-mail: amandinhacelis@gmail.com

Luciana Kelly da Silva Fonseca ORCID: https://orcid.org/0000-0001-8832-5261 Universidade Federal do Piauí, Brasil E-mail: L.kelly_fonseca@hotmail.com

Samaritana Barros do Nascimento ORCID: https://orcid.org/0000-0002-9942-4457 Universidade Federal do Piauí, Brasil

E-mail: samaritanabarros@hotmail.com

Daline da Silva Azevedo

ORCID: https://orcid.org/0000-0002-4872-2614 Universidade Federal do Delta Parnaíba, Brasil

E-mail: dalinesilvaazevedo@gmail.com

Karolaine Rodrigues Louzeiro

ORCID: https://orcid.org/0000-0002-5728-2657

Centro Universitário UNINOVAFAPI, Brasil

E-mail: karolainne97@outlook.com

João Felipe Tinto Silva

ORCID: https://orcid.org/0000-0003-3662-6673 Centro Universitário de Ciências e Tecnologia do Maranhão, Brasil

E-mail: felipetinto99@gmail.com

Rhauanna Mylena Dos Santos Castro

ORCID: https://orcid.org/0000-0001-6400-9336

Universidade Estadual do Piauí, Brasil

E-mail: rhau.castro@hotmail.com

Tito Cacau Sousa Santos

ORCID: https://orcid.org/0000-0001-7858-2301 Universidade Estadual do Piauí, Brasil E-mail: titocss@hormail.com

Nanielle Silva Barbosa

ORCID: https://orcid.org/0000-0001-5758-2011 Universidade Estadual do Piauí, Brasil

E-mail: naniellesilvabarbosa@ @otmail.com

Carlos Henrique Nunes Pires

ORCID: https://orcid.org/0000-0002-8408-0209 Faculdade Pitagoras, Brasil

E-mail: carloshenriqueenf1@gmail.com
Natalia da Silva Teixeira

ORCID: https://orcid.org/0000-0002-5879-8145

Centro Universitário UNINOVAFAPI, Brasil E-mail: silvanatalia280@gmail.com

Nágila Silva Alves

ORCID: https://orcid.org/0000-0002-1618-8111

Centro Universitário Santo Agostinho, Brasil E-mail: nglarraial@gmail.com

Kayron Rodrigo Ferreira Cunha ORCID: https://orcid.org/0000-0003-3507-3376 Universidade Federal do Delta Parnaíba, Brasil E-mail: ikayron.kr@gmail.com 


\title{
Resumo
}

Introdução: O isolamento social causado pela pandemia da COVID-19 para as pessoas vitimas do câncer, pode implicar diretamente no tratamento de forma negativa pois os mesmos necessitam de um atendimento multiprofissional de forma continua. Dessa forma, as avaliações de rotina foram adiadas, exames laboratoriais adiadas, tratamento interrompido e cirurgias canceladas. Entretanto, é necessário o isolamento social para que não haja a transmissão do Sars-Cov-2. Objetivo: objetivou-se descrever as possíveis repercussões que pode impactar negativamente na qualidade de vida de mulheres vitimas do câncer de mama durante a pandemia da COVDI-19. Método: $\mathrm{O}$ estudo trata-se de uma revisão integrativa da literatura, buscas foram idealizadas em todos os idiomas nas bases de dados: Biblioteca Virtual em Saúde (BVS) e PubMed e science direct, os descritores para os resultados foram: ((Breast câncer)) and ((COVID-19)) and ((quality of life)). Foram incluídos artigos de 2020-2021. Resultados e discussão: Estudos apontam que o câncer de mama é o quadro patológico que mais afeta a condição emocional das mulheres, pois reflete diretamente na feminilidade da mulher. Com isso, a pandemia da COVID-19 repercutiu ainda mais, devido ao atraso do tratamento, risco que a COVID-19 pode refletir para a saúde em geral dessas, deixando-as mais vulneráveis emocionalmente. Conclusões: Estudos apontaram que a pandemia da COVID-19 pode implicar na redução da qualidade de vida, pois as mesmas já são impactadas negativamente, pois o câncer de mama fere a feminilidade dessas mulheres. Sendo assim, poucos estudos foram localizados sobre a respetiva temática, mostrando assim a necessidade e relevância de mais evidência futuras.

Palavras-chave: Câncer de mama; COVID-19; Qualidade de vida.

\begin{abstract}
Introduction: The social isolation caused by the COVID-19 pandemic for people who are victims of cancer, can directly affect the treatment in a negative way, as they need continuous multidisciplinary care. Thus, routine assessments were postponed, laboratory tests postponed, treatment interrupted and surgeries cancelled. However, social isolation is necessary so that there is no transmission of Sars-Cov-2. Objective: This study aimed to describe the possible repercussions that can negatively impact the quality of life of women victims of breast cancer during the COVDI-19 pandemic. Method: The study is an integrative literature review, searches were designed in all languages in the databases: Virtual Health Library (VHL) and PubMed and science direct, the descriptors for the results were: $(($ Breast cancer $))$ and ((COVID-19)) and ((quality of life)). Articles from 2020-2021 were included. Results and discussion: Studies show that breast cancer is the pathological condition that most affects the emotional condition of women, as it directly reflects on the woman's femininity. Thus, the COVID-19 pandemic had even more repercussions, due to the delay in treatment, a risk that COVID-19 may reflect on their general health, leaving them more emotionally vulnerable. Conclusions: Studies have shown that the COVID-19 pandemic may result in reduced quality of life, as they are already negatively impacted, as breast cancer hurts the femininity of these women. Therefore, few studies were located on the respective theme, thus showing the need and relevance of further evidence in the future.
\end{abstract}

Keywords: Breast cancer; COVID-19; Quality of life.

\section{Resume}

Introducción: El aislamiento social que provoca la pandemia COVID-19 para las personas víctimas de cáncer, puede afectar directamente el tratamiento de forma negativa, ya que necesitan una atención multidisciplinar continua. Así, se pospusieron las evaluaciones de rutina, se pospusieron las pruebas de laboratorio, se interrumpió el tratamiento y se cancelaron las cirugías. Sin embargo, el aislamiento social es necesario para que no haya transmisión de Sars-Cov-2. Objetivo: Este estudio tuvo como objetivo describir las posibles repercusiones que pueden impactar negativamente en la calidad de vida de las mujeres víctimas de cáncer de mama durante la pandemia COVDI-19. Método: El estudio es una revisión integradora de la literatura, se diseñaron búsquedas en todos los idiomas en las bases de datos: Virtual Health Library (BVS) y PubMed y science direct, los descriptores de los resultados fueron: ((Breast cancer)) y (( COVID-19)) y ((calidad de vida)). Se incluyeron artículos de 2020-2021. Resultados y discusión: Los estudios muestran que el cáncer de mama es la condición patológica que más afecta la condición emocional de la mujer, ya que refleja directamente la feminidad de la mujer. Así, la pandemia de COVID-19 tuvo aún más repercusiones, debido al retraso en el tratamiento, riesgo de que el COVID-19 pueda reflejarse en su salud general, dejándolos más vulnerables emocionalmente. Conclusiones: Los estudios han demostrado que la pandemia de COVID-19 puede resultar en una reducción de la calidad de vida, ya que ya se ven afectadas negativamente, ya que el cáncer de mama daña la feminidad de estas mujeres. Por lo tanto, se localizaron pocos estudios sobre el tema respectivo, lo que demuestra la necesidad y relevancia de más evidencia en el futuro.

Palabras clave: Cáncer de mama; COVID-19; Calidad de vida.

\section{Introdução}

A pandemia da COVID-19 iniciou na Whuan na China, quando se alastrou mundial e por si manifestar de forma assintomática ou não assintomática, causando sintomas leves ou graves e levando ao óbito, (sintomas como, febre, cansaço, 
tosse, dispneia e pneumonia) e que pessoas doenças crônicas ou câncer foram mais afetadas por apresentarem maior quadro vulnerabilidade para o vírus, com isso, foi decretado pela Organização Mundial da Saúde (OMS) quarentena com intuito de prevenir o contagio (Correa et al., 2020).

Entretanto, a pandemia da COVI-19 repercutiu negativamente na qualidade de vida pessoas vulneráveis vitimas do câncer, pois o convívio social pode traze um bem-estar, consequentemente podendo impactar na interrupção do tratamento e reduzir a qualidade de vida. De acordo com a OMS a qualidade de vida implica no bem-estar físico, mental e social (Correa et al., 2020; Schandl et al., 2021; Gallo et al., 2021).

Dessa forma, o isolamento social para as pessoas vitimas do câncer, pode implicar diretamente no tratamento de forma negativa pois os mesmos necessitam de um atendimento multiprofissional de forma continua. À vista disso, a pandemia da COVID-19 pode repercutir na interrupção das avaliações de rotina, exames laboratoriais adiadas, tratamento interrompido e cirurgias canceladas. Entretanto, é necessário o isolamento social para que não haja a transmissão do Sars-Cov-2 (Mellisa et al., 2020; Jonas et al., 2021; Greco et al., 2021).

Com isso, o momento pandêmico pode ser desafiador para as pessoas que estão passando pelo tratamento oncológico. No câncer de mama as mulheres ficam com mais aflição, pois as mesmas já possuem a condição emocional vulnerável devido o quadro patológico, devido as reações do tratamento e por ferir seus aspetos de feminilidade (Papandreou et al., 2021). Dessa forma, pode gerar medo da evolução do quadro patológico devido às interrupções gerado devido o isolamento da pandemia da COVID-19 e medo de transmissão do vírus (Guven et al., 2020; Stefan et al 2021).

No entanto, o isolamento poderá levar a aproximação familiar o que repercutir de forma significativa, pois o tratamento de paciente oncológicos pode ser difícil para as mesmas, devido os efeitos colaterais do tratamento. No entanto, pode repercutir na qualidade de vida devido à interrupção do tratamento atraso no diagnostico e cirurgias adiadas. Sendo assim o estudo objetivou-se descrever as possíveis repercussões que pode impactar negativamente na qualidade de vida de mulheres vitimas do câncer de mama durante a pandemia da COVDI-19.

\section{Metodologia}

O presente estudo trata-se de uma revisão integrativa da literatura pelos quais teve como busca nos bancos de dados: Biblioteca Virtual em Saúde (BVS) e PubMed e science direct, os descritores para os resultados foram: ((Breast câncer)) and ((COVID-19)) and ((quality of life)) os mesmos foram combinados entre si e disponível de acordo com o e Descritores em Ciências da Saúde (DeCS), utilizando operador Booleano "AND”. Com o intuído de nortear os achados sobre possíveis complicações impactas da qualidade de vida de mulheres vitimas do câncer de mama em tempos da pandemia da COVID-19, as buscas foram realizadas, selecionando para elegibilidade sua relevância inicialmente por meio de títulos e resumos e caso houvesse necessidade texto completo o (Mendes; Silveira; Galvão 2008).

Para a elegibilidade foram norteadas as buscas dos artigos em todos os idiomas, incluindo-se pesquisa de publicação de 2020-2021, estudos já dispostos na literatura implicando possíveis complicações possíveis impactos da qualidade de vida de mulheres com câncer de mama e seus sinônimos, com isso incluídos apenas estudos que respondesse ao objetivo proposto.

Dessa forma, foram excluídas revisões, meta-análise, cartas ao editor, resumos, artigos de opinião, estudos de caso e teses de mestrado ou doutorado e que não respondia o objetivo proposto.

A natureza do estudo foi embasada por meio do objetivo do estudo, diferenciados naqueles que objetivaram avaliar associação às informações significativas e foram narradas e comparadas.

A pergunta norteadora foi:" A qualidade de vida de mulheres vitimas do câncer de mama podem ser impactadas negativamente durante a pandemia da COVID-19?" com uso da estratégia "PICO" representa um acrônimo para Paciente, Intervenção, Comparação e "Outcomes" (desfecho). "P” Mulheres com câncer de mama, "O" Qualidade de vida de mulheres 
com câncer de mama, "I" não se aplica por não se tratar de intervenções e "C" não se aplica por não se tratar de grupos comparativos (Tabela:1), (Santos et al., 2007).

Tabela 1. "PICO".

\begin{tabular}{|c|c|c|}
\hline Acrônimo & Descrição & Componentes da questão \\
\hline $\mathrm{P}$ & População & Mulheres com câncer de mama \\
\hline I & Interesse & - \\
\hline $\mathrm{C}$ & $\begin{array}{l}\text { Controle } \\
\text { comparação }\end{array}$ & - \\
\hline $\mathrm{O}$ & Desfecho & Qualidade de vida de mulheres com câncer de mama \\
\hline
\end{tabular}

Fonte: Autores.

Para a extração de dados considerados relevantes em cada produção os autores elaboraram formulário contendo informações sobre identificação do artigo, autoria, país e ano de publicação, abordagem metodológica, principais conclusões e nível de evidência (NE). As evidências foram classificadas conforme o modelo proposto: nível I (evidências oriundas de revisão sistemática ou metanálise de todos ensaios clínicos relevantes randomizados controlados ou provenientes de diretrizes clínicas baseadas em revisões sistemáticas de ensaios clínicos randomizados controlados); nível II (evidências derivadas de pelo menos um ensaio clínico randomizado controlado bem delineado) (Melnyk; Overholt, 2005).

Com isso, o nível III (evidências obtidas de ensaios clínicos bem delineados sem randomização); nível IV (evidências provenientes de estudos de coorte e de caso-controle bem delineados); nível V (evidências originárias de revisão sistemática de estudos descritivos e qualitativos); nível VI (evidências derivadas de um único estudo descritivo ou qualitativo); e nível VII (evidências oriundas de opinião de autoridades e/ou relatórios de comitês de especialistas) (Melnyk; Overholt, 2005).

\section{Resultados}

Foram localizados 51 estudos, porém apenas 6 estavam dentro da elegibilidade, ambos os estudos em idioma em inglês, mais informações na (Tabela 2). Tais artigos foram mencionados suas elegibilidades no (Figura 1). 
Figura 1. Distribuição dos artigos, segundo o ano de publicação, banco de dados, periódicos de publicação e abordagens.

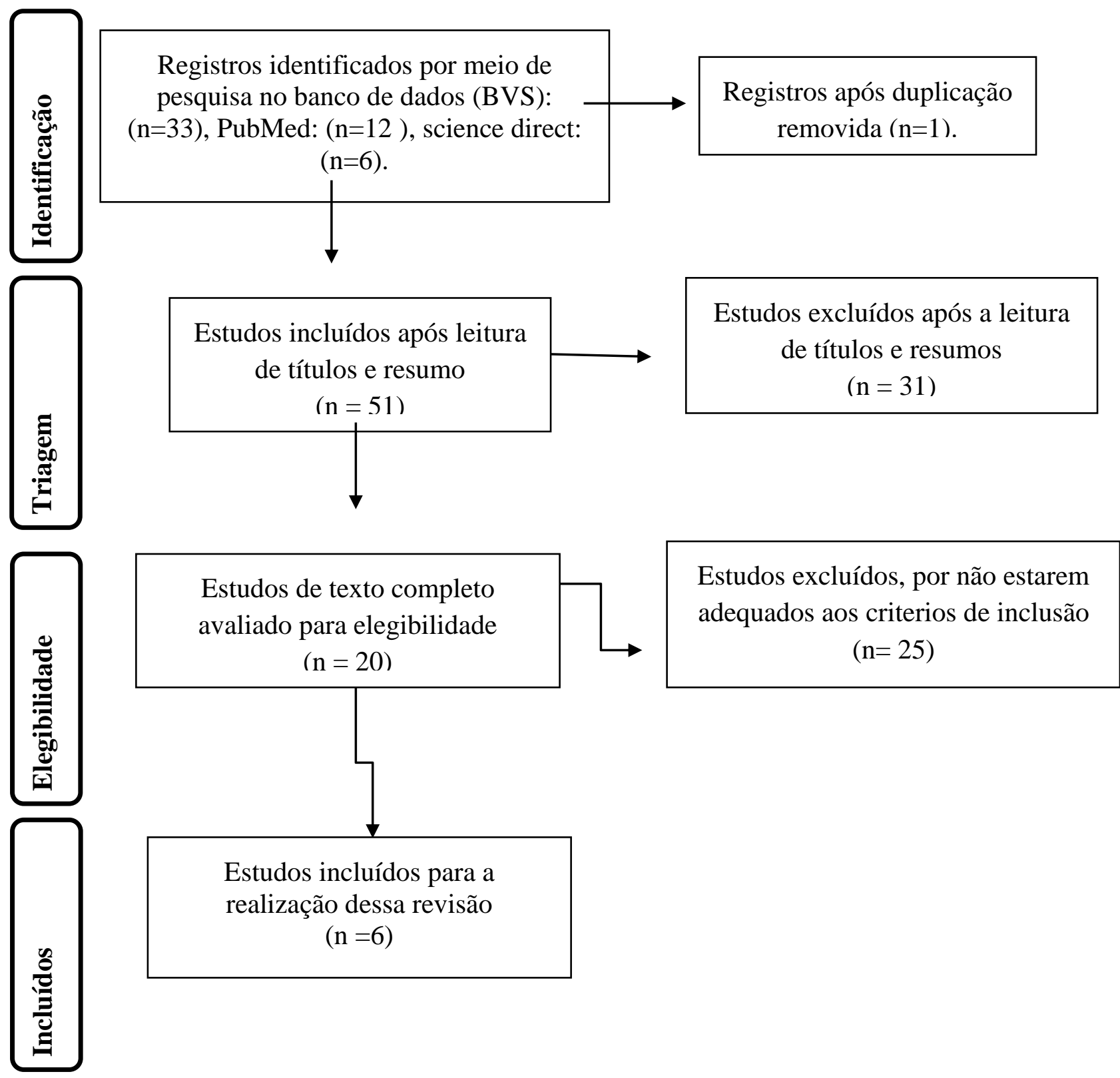

Figura 1: Resultados das buscas de 2020-2021. Fonte: www.prisma-statement.org.

Sendo assim, seis (100\%) foram incluídas para síntese. Cinco (83,3\%) foram publicados no ano de 2021, ressaltando a abordagem recente na literatura sobre o tema. Houve predomínio de publicações internacionais, 5 (83,3\%). Quanto ao NE, 4 produções $(66,6 \%)$ classificaram como nível III (evidências obtidas de ensaios clínicos bem delineados sem randomização) e duas produções $(33,3 \%)$ classificaram como nível IV (evidências provenientes de estudos de coorte e de caso-controle bem delineados). Os estudos evidenciaram que os pacientes oncológicos estão mais suscetíveis ao adoecimento devido ao atraso no diagnostico, tratamento e procedimentos necessários para a manutenção de vida durante a pandemia da Covid-19. A síntese dos achados encontra-se organizada na Tabela 2. 
Tabela 2. Sumarização das produções incluídas na síntese conforme título, autores, ano, país de publicação, abordagem metodológica, principais conclusões e NE. Teresina (PI), Brasil, 2021.

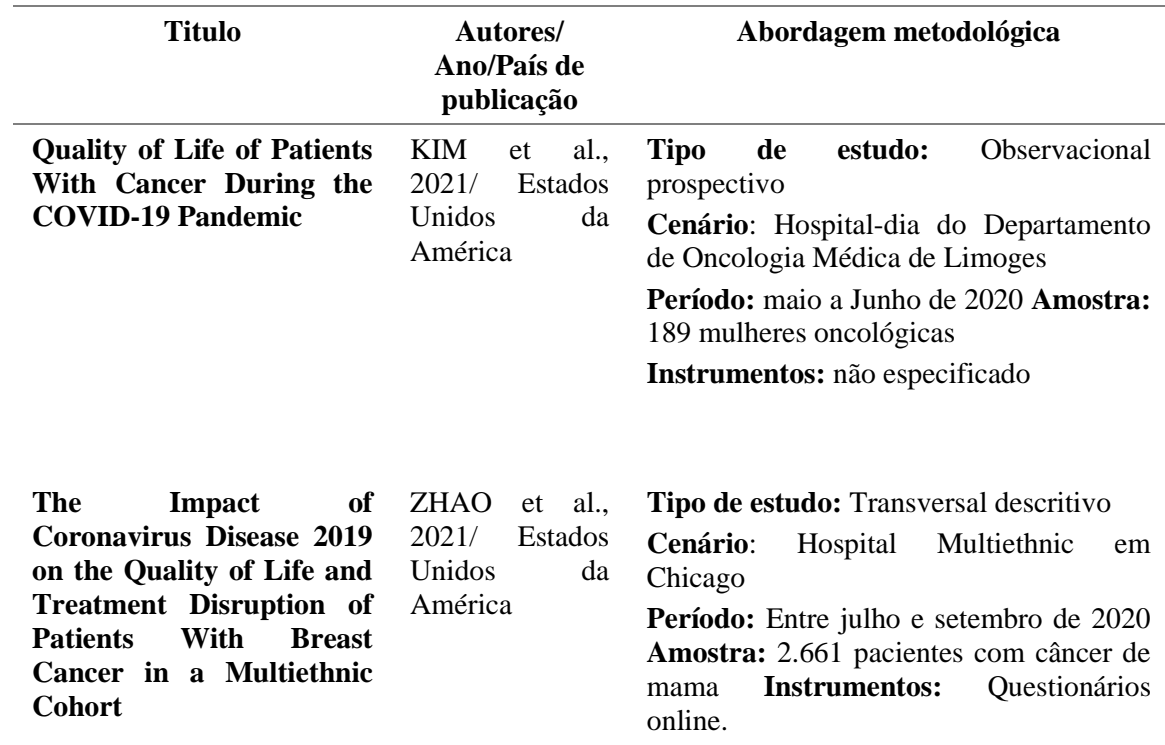

Comparative analysis: QOL in breast cancer patients before and during the COVID-19 pandemic

Economic impact of avoidable cancer deaths caused by

diagnostic delay during the COVID-19 pandemic: A

national population-based modelling study in

England, UK

Evaluating the impact of COVID-19 on supportive care needs, psychological distress and quality of life in UK cancer survivors and their support etwork
PINHOLATO et Tipo de estudo: Transversal descritivo al., 2021/ Brasil qualitativo

Cenário: Centro

de Apoio ao Paciente com Câncer de Londrina

Período: janeiro a agosto de 2020

Amostra: 185 mulheres com câncer de mama Instrumentos: Questionários online

GHEORGHE et al., 2021/ Reino Unido

Tipo de estudo: Transversal prospectivo Cenário: Centro para Análise de Doenças Infecciosas Globais de Londres

Período: março de 2020

Amostra: 32.583 pacientes com câncer de mama Instrumentos: Registro epidemiológico de câncer do Serviço Nacional de Saúde

HULBERT et Tipo de estudo: Transversal prospectivo al., 2021/ Reino Cenário: Hospital para tratamento de Unido câncer

Período: junho e julho de 2020

Amostra: 144 mulheres com câncer de mama

Instrumentos: Questionário on-line

PAPAUTSKY; HAMLISH,

Emotional Response of US Breast Cancer Survivors during the COVID-19 Pandemic

2020/ Estados

Unidos

América
Tipo de estudo: Transversal descritivo Cenário: Clinica de tratamento de câncer

Período: abril a maio de 2020

Amostra: 633 sobreviventes de câncer de mama

Instrumentos: Questionário on-line
Principais conclusões

NE

Pacientes oncológicos durante a III pandemia da COVID-19, não possuem somente comprometimentos físicos, mas também psicossociais, esses podem estar associados ao medo do não acompanhamento do estado de saúde, por outras vezes diagnósticos e tratamentos atrasados, o que pode prejudicar esses pacientes.

A vida de pacientes com câncer de mama e seus tratamentos rogramados foram adversamente afetados durante a pandemia de COVID-19. Assim mais apoio deve ser fornecido por centros hospitalares e pela comunidade de pesquisa médica para pacientes com câncer durante esta pandemia.

Mulheres diagnosticadas durante a pandemia tiveram um escore físico e emocional mais baixo em comparação com as pacientes diagnosticadas antes da pandemia do COVID-19. Demonstrando a perda de vários aspectos relacionados a qualidade de vida dessas mulheres.

Mortes prematuras por câncer resultantes de atrasos no diagnóstico durante a primeira onda da pandemia de COVID-19 no Reino Unido resultou em perdas significativas. Esse impacto é, de fato, maior do que o das mortes diretamente atribuíveis ao COVID-19.

A pandemia da COVID-19 levou a um reajuste das necessidades não atendidas em diversos domínios psicossociais e de bem-estar de pacientes oncológicos. À medida que o distanciamento social continua como estratégias de saúde pública para proteger os mais vulneráveis do COVID-19, as intervenções para apoiar as necessidades não atendidas organizadas de pacientes com câncer devem ser priorizados.

Pessoas que estão em tratamento ativo de câncer experimentam atrasos no atendimento e estão mais preocupados com

seu câncer. Além disso os relataram maior preocupação com sua saúde geral perante a COVID-19. 


\section{Discussão}

Estudos apontam que o câncer de mama é o quadro patológico que mais afeta a condição emocional das mulheres, pois reflete diretamente na feminilidade da mulher. Com isso, a pandemia da COVID-19 repercutiu ainda mais, devido ao atraso do tratamento, risco que a COVID-19 pode refletir para a saúde em geral dessas, deixando-as mais vulneráveis emocionalmente (Papautsky \& Hamlish, 2020).

Desse modo, um estudo realizado na França demostrou que a condição de ansiedade das mulheres com câncer de mama e outros como câncer de pulmão e colorretal permaneceu baixo, ou seja, a COVID-19 não impactou mais ainda na qualidade de vida, entretanto $11 \%$ desses pacientes demostraram um nível de ansiedade, devido à condição patológica e saúde física estável (Baffert et al., 2021).

No entanto, a pandemia do coronavírus, pode aproximar o convívio familiar entre as pessoas, e para os pacientes vitimas do câncer pode melhora a qualidade de vida, já que o quadro patológico em si afeta tanto a condição emocional por deixa-los mais vulneráveis emocionalmente, em um estudo com 144 mulheres vitimas do câncer de mama, relataram que reduziu de o nível de ansiedade durante o período pandêmico ( $\mathrm{p}=0,049)$ e melhorou forma significativa a qualidade de vida $(\mathrm{p}=0,032)$ no entanto pacientes em rede apoio relatou uma redução na qualidade após o inicio pandêmico $(\mathrm{p}=0,009)$ (Williams et al., 2021).

Em contrata partida, um estudo de coorte com 2.661 mulheres com câncer de mama durante o período pandêmico da COVID-19, destes, 314 foram testado positivo para o SARS-CoV-2, mostrou que a COVID-19 impactou significativamente na qualidade de vida dessas mulheres com câncer de mama, mesmas as que não testaram positivo. Dessa forma, os fatores que repercutiu negativamente na qualidade de vida foram o isolamento social que acarretou em aumento de ansiedade, condição financeira, preocupações com os desafios enfrentados para concluir o tratamento (Zhao et al., 2021).

Por consequência, a qualidade de vida de pessoas com câncer reduzio significativamente (72, 4 a 68,7, p=0,007), mostra um estudo de coorte com 318 participantes com metátese ósseo, 85\% expressão estar aflitas devido ao medo de se contaminar com o vírus do SARS-CoV-2, e outras demostrara medo com o acesso ao tratamento ( $\mathrm{n}=83,49 \%$ ) (Bartels et al., 2021).

Sendo assim, qualidade vida de pessoa com foi afeta forma global durante a pandemia da COVID-19, podendo aumentar a progressão do tumor, $20 \%$ das pessoas vitimas do câncer no momento pandêmico teve a quimioterapia adiada e 5\% abandonaram o tratamento oncológico em meio ao avanço do quadro patológico, por consequência disso, desencadeou uma perca do condicionamento físico ( $\mathrm{P}=0,088)$, emocional ( $\mathrm{P}=0,0069)$, social $(\mathrm{P}=0,0001)$, insônia $(\mathrm{P}<0,0001)$ e fadiga $(\mathrm{P}=0,003) \mathrm{e}$ perda de apetite $(\mathrm{P}=0,006)$ (Ciazynskal et al., 2020).

Dessa forma, um estudo transversal com intuito de compara à repercussão da qualidade de vida de mulheres com câncer de mama em 2014 a 2019 e com pacientes que foram diagnostica de câncer de mama em janeiro a agosto de 2020, mostrou que mulheres que foram diagnosticado com câncer de mama em 2020 tiveram a qualidade vida impactada negativamente em comparação as mulheres em diagnosticadas em 2014 a 2019 (Pinholato et al., 2021). Dessa forma, a pandemia da COVID-19 pode repercutir na vulnerabilidade de condição emocional, podendo desencadear níveis de ansiedade e depressão (Yildirim et al., 2021).

Com isso, a pandemia da COVID-19 repercutiu negativamente em morte prematura de mulheres com câncer mama, devida o atraso do diagnostico resultante da primeira onda do período pandêmico. Nos estado Unidos após a primeiro onda do vírus da COVID-19 proximamente 3 milhões de pessoas incluído homens e mulheres, não passaram pela investigação da triagem devido o isolamento social que repercutiu na suspensão de assistência prestadas nas redes hospitalares (Gheorghe et al.,2021). 


\section{Considerações Finais}

Dessa forma, os estudos apontados mostraram que a pandemia da COVID-19 repercutiu negativamente na qualidade de vida de mulheres com câncer de mama, implicando na interrupção dos tratamentos, atraso das cirurgias e adiando o diagnostico.

Desse modo, a pandemia da COVID-19 pode implicar na redução da qualidade de vida, pois as mesmas já são impactadas negativamente, pois o câncer de mama fere a feminilidade dessas mulheres. Sendo assim, poucos estudos foram localizados sobre a respetiva temática, mostrando assim a necessidade e relevância de mais evidência futuras.

\section{Referências}

Baffrt, K. A. et al. Quality of Life of Patients With Cancer During the COVID-19 Pandemic. in vivo. 35: 663-670(2021) 10.21873/invivo.12306.

Bojnrodi, T. E. et al., (2021) A case-control study to predict the risk of gestational diabetes mellitus by initial fasting blood sugar or past gestational history. International Journal of Reproductive BioMedicine 19(4), org/10.18502/ijrm.v19i4.9064.

Corrêa, K. M. et al. Impacto na Qualidade de Vida de Pacientes com Câncer em meio à Pandemia de Covid-19: uma Reflexão a partir da Teoria das Necessidades Humanas Básicas de Abraham Maslow. Revista Brasileira de Cancerologia, 2020; 66:e-1068. https://doi.org/10.32635/21769745.RBC.2020v66nTemaAtual.1068.

Gallo, O. et al. The impact of the COVID-19 pandemic on the quality of life of head and neck cancer survivors. Supportive Care in Cancer. https://doi.org/10.1007/s00520-021-06198-6.

Greco, F. et al. Impact of COVID-19 Pandemic on Health-Related Quality of Life in Uro-oncologic Patients: What Should We Wait For? uly 16, 2020, https://doi.org/10.1016/j.clgc.2020.07.008. American Journal of Obstetrics \& Gynecologylo, 19(2), e63-e68;

Guven D. C. et al. Perspectives, Knowledge, and Fears of Cancer Patients About COVID-19. Frontieres in oncologi., https://doi.org/10.3389/fonc.2020.01553

Jonas, K. et al. The impact of the Covid-19 pandemic on quality of life in skin cancer patients. PLOS ONE, https://doi.org/10.1371/journal.pone.0255501 August 18, 2021.

Melissa, K. et al. Impact of the coronavirus disease 2019 pandemic on the quality of life for women with ovarian câncer. Research Gynecology| https://doi.org/10.1016/j.ajog.2020.06.049, 223(5), P725.e1-725.e9.

Melnyk, B. M.; Overholt, E. Making the case for evidence-based practice. In: Evidence-based practice in nursing \& healthcare: a guide to best practice. Philadelphia: Lippincot Williams \& Wilkins.

Mendes, K. D. S; Silveira, R. C. C. P.; Galvão, C.M. Revisão integrativa: método de pesquisa para a incorporação de evidências na saúde e na enfermagem. Texto \& contexto enferm. 17(4), 758-64.

Month, O et al.The Impact of Coronavirus Disease 2019 on the Quality of Life and Treatment Disruption of Patients With Breast Cancer in a Multiethnic Cohort. $10.1002 /$ cncr.33798.

Papandreou et al. Application of Clinical Decision Support System to Assist Breast Cancer Patients with Lifestyle Modifications during the COVID-19 Pandemic: A Randomised Controlled Trial. Nutrients 2021, 13, 2115. https://doi.org/10.3390/nu13062115

Papautsky \& Hamlish. Emotional Response of US Breast Cancer Survivors during the COVID-19 Pandemic. Cancer Investigation 2021, 39(1), 3-8 https://doi.org/10.1080/07357907.2020.1841220.

Pinholato, L. A. et al. Comparative analysis: QOL in breast cancer patients before and during the COVID-19 pandemic. Mastology 2021;31:e20200084. https://doi.org/10.29289/2594539420200084.

Santos, C. M. C. et al. The Pico Strategy For The Research Question Construction And Evidence Search, Rev Latino-am Enfermagem 5(3):508-11. $10.1590 /$ S0104-11692007000300023

Schandl, et al. Social distancing during the COVID-19 pandemic and health-related quality of life among esophageal cancer survivors. Acta Oncologica, 60:8, 1011-1018, 2021, 10.1080/0284186X.2021.1921260.

Stefan, $\mathrm{S}$ et al. Quality of life in patients with cancer during the COVID-19 pandemic - a Danish cross-sectional study (COPICADS). Acta Oncologica, 60:1, 4-12, 2021. 10.1080/0284186X.2020.1830169.

Travert, C. et al. COVID-19 et cancer bronchique: adaptation des schémas d'immunothérapie et qualité de vieImmunotherapy adaptation in lung cancer during the COVID-19 pandemic. Revue des Maladies Respiratoires, https://doi.org/10.1016/j.rmr.2021.06.005Get rights and content

Williams, N. J. et al. Evaluating the impact of COVID- 19 on supportive care needs, psychological distress and quality of life in UK cancer survivors and their support network. 10.1111/ecc.13442. Eur J Cancer Care ;00:e13442.

Yildirim, O. A. et al. Depression and anxiety in cancer patients before and during the SARS-CoV-2 pandemic: association with treatment delas. Quality of Life Research (2021) 30:1903-1912 https://doi.org/10.1007/s11136-021-02795-4. 